\title{
Relation between selectivity and conductivity in narrow ion channels
}

\author{
D. G. Luchinsky \\ SGT Inc., Greenbelt \\ MD, 20770, USA \\ Email: dmitry.g.luchinsky@nasa.gov
}

\author{
W.A.T. Gibby \\ I.Kh. Kaufman \\ and P.V.E. McClintock \\ Department of Physics \\ Lancaster University \\ Lancaster LA1 4YB, UK
}

\author{
D.A. Timucin \\ Ames Research Center \\ Moffett Field \\ CA, 94035, USA
}

\begin{abstract}
To establish the general statistical mechanical properties of highly conductive but selective nano-filters we develop an equilibrium statistical-mechanical theory of the KesA filter, find the probabilities for the filter to bind ions from the mixed intra- and extra-cellular solutions, and evaluate the conductivity of the filter in its linear response regime. The results provide first principles analytical resolution of the long-standing paradox how can narrow filter conduct potassium ions at nearly the rate of free diffusion while strongly selecting them over sodium ions - and are applicable to a wide range of biological and artificial channels.
\end{abstract}

\section{INTRODUCTION}

Ion channels are located within the membrane of most cells and can conduct ions at very high rate with input of metabolic energy. Their malfunction may have disastrous consequences for the organism. Recent studies of ion channels have unravelled many secrets of their structure-function relationship, but their ability to conduct larger ions at almost the free diffusion rate, while discriminating strongly against smaller ions of the same valence remains at the centre of a "many-voiced debate" [1], [2], [3] over decades [2], [3], [4], [5], [6], [7], [8], [9], [10], [11], [12], [13].

Let us consider e.g. the potassium channel's ability [6], [8], [9] to conduct up to $\sim 10^{8} \mathrm{~K}^{+}$ions per second, while discriminating strongly against the smaller $\mathrm{Na}^{+}$ion. A widelyaccepted explanation of this extraordinary phenomenon is based on the idea of a "snug fit" [4], [6] and an iso-energetic pathway [6], [8], [2] for $\mathrm{K}^{+}$, whereas a $\mathrm{Na}^{+}$ion is confronted by a large free energy barrier. The latter is defined through the Eisenman [5] relation

$$
\Delta \Delta \bar{\mu}_{K, N a}=\left(\bar{\mu}_{N a}^{c}-\bar{\mu}_{N a}^{b}\right)-\left(\bar{\mu}_{K}^{c}-\bar{\mu}_{K}^{b}\right),
$$

where we use $\Delta \bar{\mu}_{i}^{b}=\bar{\mu}_{i}^{b}-\bar{\mu}_{i}^{c}$ to define the change in excess chemical potential during the transition of an ion of the $i$-th type from the bulk (b) to the channel (c).

However, this picture reflects neither the observed highaffinity of $\mathrm{K}^{+}$binding sites, nor multi-ion nature of the transition mechanism [9], [14], nor the reality of flexible channel walls [15], [3]. It was also argued [3] that this picture can be self-contradictory because, if an ion binds to the channel too tightly, it cannot move rapidly through it. The assumption was that the solution of the problem might require the development of a multi-particle non-equilibrium theory [3], [16] to encompass possible conformational changes and the "knock-on" mechanism of conduction [9], [14], [10], [17].

Theoretical analysis of this problem has benefited from a number of important contributions including e.g. statistical theory of selective ion channels [18], [19], [20], theory of transport in one-dimensional Coulomb gases [21], [22], theory of single-file multi-particle diffusion in narrow pores [23], [24], theory of ion selectivity and permeation [25], [26], [27], [28], theory of ion conductivity in trans-membrane channels [29], non-equilibrium thermodynamics of ion channels [30], and Coulomb blockade [31], [32], [33], [34] and dehydration theory [35] of channels to mention a few.

Despite all this progress the relationship between selectivity and conductivity in these channels remains elusive[2], [3].

Here we present an equilibrium statistical theory that describes key properties of the KcsA selectivity filter including its conductivity, the "snug fit" and "knock-on" conditions, and the Eisenman selectivity relations. A first-principles resolution of the above mentioned long-standing paradox is obtained by deriving an effective grand canonical ensemble of the filter that leads to an analytical expression for its conductivity and by showing that the Eisenman-type selectivity relation for ions of equal valence follows from the condition of diffusion-limited conductivity.

We consider the KcsA filter to be a narrow tunnel of length $12 \AA$ lined with 20 oxygen atoms providing four binding sites, numbered $S_{1}-S_{4}$ from the extracellular to intracellular side [7], [8], [9], [2], [10], [11], [12], [13], [3], [14], [15], [16], [20], (inset of Fig. 1). Under physiological conditions, this filter contains two resident $\mathrm{K}^{+}$ions separated by a water molecule. We consider conduction through the $\mathrm{K}^{+}$selectivity filter immersed in bath solutions containing a mixture of different ions. Conduction occurs when a third ion enters, knocking-on [14], [10], [17], [23] the ion on the opposite side.

\section{THEORY}

To derive conditions for the latter conduction mechanism we write the current density of the filter $j_{i}$ as [36], [37], [38] 


$$
j_{i}=-\frac{\sigma_{i}}{q} \nabla\left(k T \ln \left(c_{i} / c_{0}\right)+\bar{\mu}_{i}+q \phi\right),
$$

Here $q$ is the unit charge, $c_{i}$ are the number densities, $c_{0}$ is the normalization constant, $T$ is the temperature, $\phi$ is the electrostatic potential, and $k T \ln \left(c_{i} / c_{0}\right)+\bar{\mu}_{i}+q \phi$ is the electrochemical potential $\mu_{i}$ of the ions in the filter.

We show [39] that conductivity $\sigma_{i}$ of narrow filters for coordinated single-file motion of ions is given by generalized Einstein relation [40]

$$
\sigma_{i}=q^{2} D_{i} \partial c_{i} / \partial \eta_{i} \propto-T\left(\partial^{2} \Omega / \partial \eta_{i}^{2}\right)_{T, V},
$$

here $D_{i}$ is the chemical diffusion coefficient and $\eta_{i}$ is the chemical potential of the ions. To find the grand potential $\Omega$ we derive [39] the free energy of the system's state in the form

$$
\begin{array}{r}
G\left(\left\{n_{j}\right\}, n_{f}\right)=-k T \sum_{i} n_{i} \ln x_{i}^{b}-\sum_{i} n_{i} \Delta \widetilde{\mu}_{i}^{b}+ \\
k T \sum_{i} \ln n_{i} !+\varepsilon\left(\left\{n_{j}\right\}, n_{f}\right) .
\end{array}
$$

Here, $x_{i}^{b}$ and $n_{i}$ are the mole fraction numbers of ions of the $i$-th type in the bulk and in the filter, $\Delta \widetilde{\mu}_{i}^{b}=\Delta \bar{\mu}_{i}^{b}+q \Delta \phi^{b}$ is the difference in excess chemical and electrostatic potentials between ions in the bulk and in the channel, $\varepsilon\left(\left\{n_{j}\right\}, n_{f}\right)$ is the correlation corrections [21], [22], [33], [41] to the energy of the state due to ion-ion and ion-wall-charge interactions, and $n_{f}$ is determined by the total fixed charge on the filter's wall, $Q_{f}=q z_{f} n_{f}$. Here we defined [39] the state of the system by the set of numbers of ions of each type present in the filter $\left\{n_{j}\right\}=\left\{n_{1}, n_{2}, \ldots, n_{M}\right\}$.

The grand canonical ensemble of the filter can now be written as [39]

$$
\begin{aligned}
& P\left(\left\{n_{j}\right\}, n_{f}\right)=\mathcal{Z}^{-1}\left(\prod_{i=1}^{m} \frac{\left(x_{i}^{b}\right)^{n_{i}}}{n_{i} !}\right) \times \\
& \exp \left(\frac{1}{k T}\left(\sum_{i} n_{i}\left(\Delta \widetilde{\mu}_{i}^{b}\right)-\varepsilon\left(\left\{n_{j}\right\}, n_{f}\right)\right)\right),
\end{aligned}
$$

The effective grand partition function $Z$ is defined by the normalization condition for $P\left(\left\{n_{i}\right\}, n_{f}\right)$ and the grand potential is: $\Omega=-k T \ln Z$. The analytic forms of the probabilities $P\left(\left\{n_{i}\right\}, n_{f}\right)$ and the effective grand partition function can be related [39] to those obtained [18], [19], [20] in the form of configuration integrals.

The theory that we introduce relates $\sigma_{i}$ to key parameters of the system such as the mole fraction of conducting ions in the bulk solutions, the difference in excess chemical potential between the bulk and the filter, the fixed charge on the filter's wall, the filter's geometry, and the energy of the multi-particle interactions in the filter. This in turn allows us to establish explicit analytical relationships between filter's conductivity and selectivity.

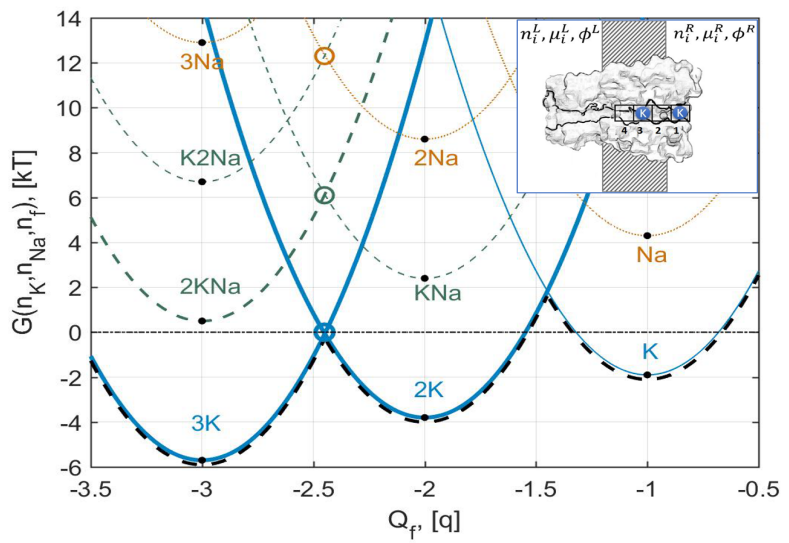

Fig. 1. Levels of free energy as a function of $Q_{f}$ for $\mathrm{K}^{+}$(blue lines), $\mathrm{Na}^{+}$ (orange dotted lines) and mixed (green dashed lines) conduction as given by Eq. (4) for the following parameters: $\Delta \bar{\mu}_{K}=2 k T$; and $\Delta \bar{\mu}_{N a}=-4.3 k T$; $U_{c} \approx 18.7 k T$. Black dots show positions of the energy level minima; the ground state is indicated by the bold black-dashed line; and open circles show locations of the resonant conduction. The labels at the origin of each parabola indicate the number of ions of each type in the filter. The inset shows schematic representation of the selectivity filter coupled to bath solutions. The binding sites $S_{1}-S_{4}$ for cations are indicated by black squares.

To calculate the conductivity (3) we use $\left\langle\Delta n_{i}^{2}\right\rangle=$ $-k T \partial \Omega / \partial \eta_{i}$, the mean number of particles and their variance in the form [42]

$$
\begin{gathered}
\left\langle n_{i}\right\rangle=\sum_{\left\{n_{i}\right\}} n_{i} P\left(n_{i}, n_{f}\right), \quad\left\langle n_{i}^{2}\right\rangle=\sum_{\left\{n_{i}\right\}} n_{i}^{2} P\left(n_{i}, n_{f}\right), \\
\left\langle\Delta n_{i}^{2}\right\rangle=\left\langle n_{i}^{2}\right\rangle-\left\langle n_{i}\right\rangle^{2}
\end{gathered}
$$

\section{ApPlicAtion to KCSA CHANNEL}

To find the free energy in each state of the KcsA filter we consider only $\mathrm{K}^{+}$and $\mathrm{Na}^{+}$ions as conducting species and limit the maximum total number of ions in the filter to three. The free energy (4) of the resultant nine system states containing non-zero numbers of ions is shown in Fig. 1. Here the correlation corrections $\varepsilon\left(n_{K}, n_{N a}, n_{f}\right)$ were approximated by the filter's dielectric barrier energy [22], [39], [43]

$$
\varepsilon\left(n_{K}, n_{N a}, n_{f}\right)=U_{c}\left(n_{K}+n_{N a}+z_{f} n_{f}\right)^{2} .
$$

The energy minimum of each parabola corresponds to the most probable combination of ions in the filter, given by the condition

$$
n_{K}+n_{N a}=-z_{f} n_{f}=1,2 \text {, or } 3 .
$$

The lowest levels, shown by solid blue lines, correspond to the filter being filled with one, two, or three $\mathrm{K}^{+}$ions, respectively. The highest energy levels (orange dotted lines) correspond to system states with $\mathrm{Na}^{+}, 2 \mathrm{Na}^{+}$, and $3 \mathrm{Na}^{+}$. The mixed states of the filter filled with $\mathrm{K}^{+}$and $\mathrm{Na}^{+}$ions are of intermediate energy as shown by green dashed lines. The shift of the energy levels for each type of ions is controlled by $\Delta \mu_{i}$.

The condition for barrier-less diffusion-limited conduction is given by [39] (cf [41], [32], [44], [23])

$$
G\left(n_{K}+1, n_{f}\right)=G\left(n_{K}, n_{f}\right) \approx 0
$$


For the total charge on the filter's wall $Q_{f} \approx-2.5 q$ and $\Delta \bar{\mu}_{K}=2 k T$ the barrier-less "knock-on" mechanism of pure $\mathrm{K}^{+}$conduction $\left(2 \mathrm{~K}^{+} \leftrightarrow 3 \mathrm{~K}^{+}\right)$corresponds to the minimum energy in the system.

Fig. 2 presents the filter's conductivity/selectivity relations for potassium and sodium ions as a function of the key filter parameters $\Delta \mu_{K}$ and $Q_{f}$. It can be seen in the figure that for small absolute values of $\Delta \mu_{K}<0.5$ (left-hand side of figure) the filter is non-selective and both conductivities $\sigma_{K}$ and $\sigma_{N a}$ are relatively large for any value of the wall charge $Q_{f}$. With $\Delta \mu_{K}$ increasing above 1 the free energy for sodium to enter the channel becomes $\Delta G_{N a}>5 k T\left(\Delta \bar{\mu}_{N a}=-4.3 k T\right)$, the filter's conductivity becomes strongly selective for $\mathrm{K}^{+}$ ions. The increasing affinity of $\mathrm{K}^{+}$ions results in a strong attenuation of the $\sigma_{K}$ as was conjectured earlier [3]. However, narrow bands of high conductivity remain, similar to those observed for divalent ions in Brownian dynamics modeling of calcium channel [41], [32]. The width of these conductivity bands is $\sim 1 / U_{c}$. Therefore, simultaneous strong selectivity and high conductivity of the channel can be achieved only at the locations of the peaks of $\sigma_{K}$.

To derive an exact conductivity/selectivity relationship we write the potential barrier for a $\mathrm{K}^{+}$ion to enter the channel when occupied by two potassium ions in the form $\Delta G_{K}=G\left(3 K, 0, n_{f}\right)-G\left(2 K, 0, n_{f}\right)$; and compare it with the barrier for a sodium ion to enter the channel $\Delta G_{N a}=$ $G\left(2 K, N a, n_{f}\right)-G\left(2 K, 0, n_{f}\right)$.

Solving [39] the Eqs. (8) with respect to optimal values of $n_{f}^{*}=\left(z_{f} n_{f}\right)^{*}$ and $\Delta \mu_{K}^{*}$ we have

$$
\begin{array}{r}
n_{f}^{*}=-\left(n_{K}+\frac{1}{2}\right)+\frac{C}{q^{2}}\left(k T \ln \left(n_{K}+1\right)-\Delta \mu_{K}^{*}\right), \\
\Delta \mu_{K}^{*}=\frac{1}{n_{K}} \varepsilon\left(n_{K}, 0, n_{f}^{*}\right)-\frac{k T}{n_{K}} \ln n_{K} ! .
\end{array}
$$

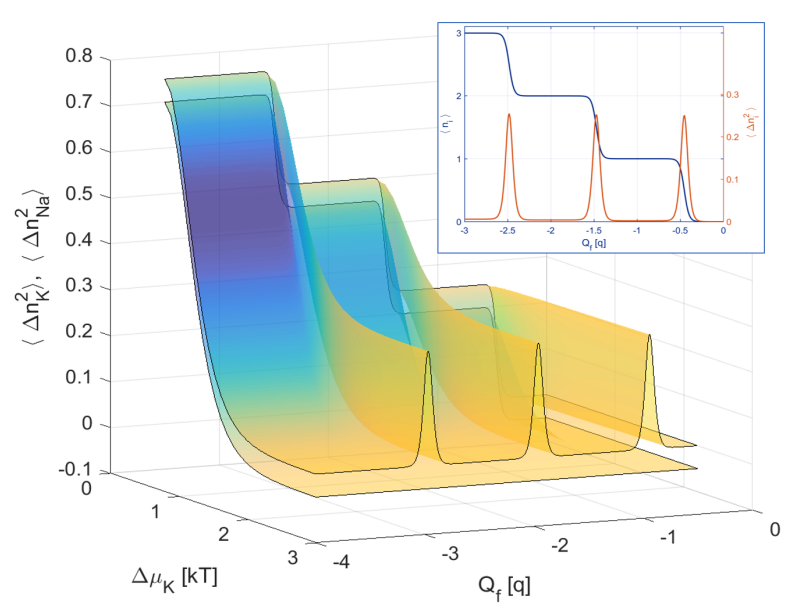

Fig. 2. Conductivity of $\mathrm{K}^{+}$(top sheet) and $\mathrm{Na}^{+}$(bottom sheet, shifted down by 0.05$)$ as a function of the system parameters $\Delta \mu_{K}$ and $Q_{f}$. When $\Delta \mu_{K} \simeq$ $0 k T$ the conductivities are nearly same. With increasing $\Delta \mu_{K}$, however, conductivity becomes increasingly selective: sharp ridges corresponding to conductivity bands for $\sigma_{K}$ as a function of $Q_{f}$ are clearly evident, and $\sigma_{N a} \approx$ 0 for all $Q_{f} . \Delta \mu_{N a} \simeq-4.3 k T$ for this figure.
With the resultant optimal values of $n_{f}^{*}=\left(z_{f} n_{f}\right)^{*}$ and $\Delta \mu_{K}^{*}$ given by Eqs. (9), the filter allows for the unimpeded (diffusion-limited) barrier-less $\Delta \Delta G_{K} \approx 0$ passage of potassium ions, while selectively blocking the entry of $\mathrm{Na}^{+}$ions. The barrier $\Delta \Delta G_{N a}$ for sodium ions can now be immediately derived leading to the well-known Eisenman result

$$
\begin{aligned}
& \Delta \Delta G_{N a}=\left(\bar{\mu}_{N a}^{c}-\bar{\mu}_{N a}^{b}\right)-\left(\bar{\mu}_{K}^{c}-\bar{\mu}_{K}^{b}\right)+ \\
& k T \ln \frac{c_{K}}{3 c_{N a}}=\Delta \Delta \bar{\mu}_{K, N a}+k T \ln \frac{c_{K}}{3 c_{N a}} .
\end{aligned}
$$

To derive conductivity of potassium ions we use eqs. (3)-(6) and obtain [39]

$$
\sigma_{K}=q^{2} D_{K} e^{-\Delta G_{K} / k T} / V_{c} k T\left(1+e^{-\Delta G_{K} / k T}\right)^{2},
$$

where $V_{c}$ is the volume of the filter.

\section{CONCLUSIONS}

We conclude, that Eqs. (10) and (11) resolve the selectivity $v s$. conductivity paradox. It can be seen from this expression [39] that the conductivity of the filter has a very sharp peak at the resonant transition point $2 K^{+} \leftrightarrow 3 \mathrm{~K}^{+}$, where $\Delta G_{K} \approx 0$, approaching the diffusion limit as illustrated in Fig. 2 . The proposed approach allows one to obtain analytically the key properties of the KcsA filter including its conductivity, the Eisenman selectivity relations, and the snug fit and knockon conditions. It also provides independent first-principles estimates of the values of the filter parameters $\Delta \mu_{N a}, \Delta \mu_{K}$, and of $Q_{f}$, as functions of channel structure required for potassium filter to function in accordance with experimental observations.

Estimates based on Eqs. (9)-(11) and realistic filter parameters [39], [45] show that the value of the fixed charge on the filter wall is $Q_{f} \approx-2.5 q$, the partial valence of the oxygen atoms is $z_{f} \approx 0.125$, the excess chemical potentials are $\bar{\mu}_{K}=2 k T$; and $\Delta \bar{\mu}_{N a}=-4.3 k T$, and a flow rate through the filter is of the order $\sim 10^{8}$ ions per second for a potential difference of $50 \mathrm{mV}$, while filter is discriminating 1:1000 against the (smaller) $\mathrm{Na}^{+}$ion. These values are consistent with the experimental observations reported by MacKinnon and other authors.

Finally, we comment that the results obtained are applicable far beyond the KcsA channel, and may be expected to describe conduction in a wide class of narrow, ion-selective, biological and artificial nano-filters [34], [46].

\section{ACKNOWLEDGMENT}

We are grateful to Miroslav Barabash, Bob Eisenberg, Olena Fedorenko, Carlo Guardiani, Igor Khovanov, Stephen Roberts and Aneta Stefanovska for helpful discussions. The research was supported by the Engineering and Physical Sciences Research Council UK (grant No. EP/M015831/1). 


\section{REFERENCES}

[1] J. Zheng and M. C. Trudeau, Eds., Handbook of Ion Channels. Boca Raton, FL: CRC Press Taylor \& Francis Group, 2015.

[2] K. N. Piasta, D. L. Theobald, and C. Miller, "Potassium-selective block of barium permeation through single KcsA channels." J. Gen. Physiol., vol. 138, no. 4, pp. 421-36, 2011.

[3] R. Horn, B. Roux, and J. Åqvist, "Permeation Redux: Thermodynamics and Kinetics of Ion Movement through Potassium Channels," Biophys. J., vol. 106, no. 9, pp. 1859-1863, 2014.

[4] L. J. Mullins, "An analysis of conducance changs in SQUID axon," $J$. Gen. Physiol., vol. 42, no. 5, pp. 1013-1035, 1959.

[5] G. Eisenman, "On the elementary origin of equilibrium ion specificity," in Symposium on Membrane Transport and Metabolism, A. Kleinzeller and A. Kotyk, Eds. Academic Press, New York, 1961, pp. 163-179.

[6] F. Bezanilla and C. M. Armstrong, "Negative conductance caused by entry of sodium and cesium ins into the potassium channels of squid axons," J. Gen. Physiol., vol. 60, no. 5, pp. 588-608, 1972.

[7] G. Eisenman and R. Horn, "Ionic selectivity revisited: the role of kinetic and equilibrium processes in ion permeation through channels," J. Membrane Biol., vol. 76, no. 3, pp. 197-225, 1983.

[8] D. A. Doyle, J. M. Cabral, R. A. Pfuetzner, A. Kuo, J. M. Gulbis, S. L. Cohen, B. T. Chait, and R. MacKinnon, "The structure of the potassium channel: Molecular basis of $\mathrm{K}^{+}$conduction and selectivity," Science, vol. 280, no. 5360, pp. 69-77, 1998.

[9] J. H. H. Morais-Cabral, Y. Zhou, and R. MacKinnon, "Energetic optimization of ion conduction rate by the $\mathrm{K}^{+}$selectivity filter," Nature, vol. 414, no. 6859, pp. 37-42, 2001.

[10] S. Y. Noskov and B. Roux, "Importance of hydration and dynamics on the selectivity of the KcsA and NaK channels," J. Gen. Physiol., vol. 129, no. 2, pp. 135-143, 2007.

[11] P. D. Dixit, S. Merchant, and D. Asthagiri, "Ion selectivity in the KcsA potassium channel from the perspective of the ion binding site," Biophys. J., vol. 96, no. 6, pp. 2138-2145, jan 2009.

[12] B. Roux, S. Bernèche, B. Egwolf, B. Lev, S. Noskov, C. N. Rowley, and H. Yu, "Ion selectivity in channels and transporters," J. Gen. Physiol., vol. 137, no. 5, pp. 415-426, 2011.

[13] P. D. Dixit and D. Asthagiri, "Perspectives on: Ion selectivity: Thermodynamics of ion selectivity in the KcsA K ${ }^{+}$channel," J. Gen. Physiol., vol. 137, no. 5, pp. 427-433, 2011.

[14] Y. Zhou and R. MacKinnon, "The occupancy of ions in the $\mathrm{K}^{+}$ selectivity filter: Charge balance and coupling of ion binding to a protein conformational change underlie high conduction rates," J. Mole. Biol., vol. 333, no. 5, pp. 965-975, 2003.

[15] T. W. Allen, O. S. Andersen, and B. Roux, "On the Importance of Atomic Fluctuations, Protein Flexibility, and Solvent in Ion Permeation," J. Gen. Physiol., vol. 124, no. 6, pp. 679-690, 2004.

[16] M. G. Derebe, D. B. Sauer, W. Zeng, A. Alam, N. Shi, and Y. Jiang, "Tuning the ion selectivity of tetrameric cation channels by changing the number of ion binding sites." Proc. Nat. Acad. Sci. USA, vol. 108, no. 2, pp. 598-602, 2011.

[17] P. H. Nelson, "A permeation theory for single-file ion channels: Oneand two-step models," J. Chem. Phys., vol. 134, pp. 165 102-165114, 2011.

[18] B. Roux, "Statistical mechanical equilibrium theory of selective ion channels," Biophys. J., vol. 77, no. 1, pp. 139-153, 1999.

[19] B. Roux, T. Allen, S. Berneche, and W. Im, "Theoretical and computational models of biological ion channels," Quart. Rev. Biophys., vol. 37, no. 1, pp. 15-103, 2004.

[20] B. Roux, "Ion binding sites and their representations by reduced models," Journal of Physical Chemistry B, vol. 116, no. 23, pp. 6966-6979, 2012.

[21] A. Kamenev, J. Zhang, A. I. Larkin, and B. I. Shklovskii, "Transport in one-dimensional Coulomb gases: From ion channels to nanopores," Physica A, vol. 359, no. 0, pp. 129-161, 2006.

[22] J. Zhang, A. Kamenev, and B. I. Shklovskii, "Conductance of ion channels and nanopores with charged walls: A toy model," Phys. Rev. Lett., vol. 95, no. 14, p. 148101, 2005.

[23] S. O. Yesylevskyy and V. N. Kharkyanen, "Barrier-less knock-on conduction in ion channels: peculiarity or general mechanism?" Chem. Phys., vol. 312, pp. 127-133, 2005.

[24] V. N. Kharkyanen, S. O. Yesylevskyy, and N. M. Berezetskaya, "Approximation of super-ions for single-file diffusion of multiple ions through narrow pores," Phys. Rev. E, vol. 82, p. 051103, 2010.
[25] D. Gillespie, W. Nonner, D. Henderson, and R. S. Eisenberg, "A physical mechanism for large-ion selectivity of ion channels," Phys. Chem. Chem. Phys., vol. 4, no. 19, pp. 4763-4769, 2002.

[26] D. Boda, D. Gillespie, W. Nonner, D. Henderson, D. Busath, and B. Eisenberg, "Effects of dielectrics on selectivity: Computing induced charge in Monte Carlo simulations," Biophys. J., vol. 86, no. 1, pp. 6A-6A, 2004

[27] D. Krauss, B. Eisenberg, and D. Gillespie, "Selectivity sequences in a model calcium channel: role of electrostatic field strength," Eur. Biophys. J., vol. 40, no. 6, pp. 775-782, 2011.

[28] B. Eisenberg, Y. Hyon, and C. Liu, "Energy variational analysis of ions in water and channels: Field theory for primitive models of complex ionic fluids," J. Chem. Phys., vol. 133, no. 10, p. 104104, 2010.

[29] D. M. Rogers, T. L. Beck, and S. B. Rempe.

[30] E. Kitzing, "A novel model for saturation of ion conductivity in transmembrane channels," in Membrane Proteins: Structures, Interactions and Models: Proceedings of the Twenty-Fifth Jerusalem Symposium on Quantum Chemistry and Biochemistry Held in Jerusalem, Israel, May 18-21,1992, A. Pullman, J. Jortner, and B. Pullman, Eds. Dordrecht: Springer Netherlands, 1992, pp. 297-314.

[31] M. Krems and M. Di Ventra, "Ionic Coulomb blockade in nanopores," J. Phys. Condens. Matter, vol. 25, p. 065101, 2013.

[32] I. K. Kaufman, D. G. Luchinsky, R. Tindjong, P. V. E. McClintock, and R. S. Eisenberg, "Energetics of discrete selectivity bands and mutationinduced transitions in the calcium-sodium ion channels family," Phys. Rev. E, vol. 88, no. 5, p. 052712, 2013.

[33] I. K. Kaufman, P. V. E. McClintock, and R. S. Eisenberg, "Coulomb blockade model of permeation and selectivity in biological ion channels," New J. Phys., vol. 17, no. 8, p. 083021, 2015.

[34] J. Feng, K. Liu, M. Graf, D. Dumcenco, A. Kis, M. Di Ventra, and A. Radenovic, "Observation of ionic Coulomb blockade in nanopores," Nature Mater, vol. 15, no. 8, pp. 850 - 855, 2016.

[35] M. Zwolak, J. Lagerqvist, and M. Di Ventra, "Quantized ionic conductance in nanopores," Phys. Rev. Lett., vol. 103, p. 128102, 2009.

[36] D. Gillespie, W. Nonner, and R. S. Eisenberg, "Coupling PoissonNernst-Planck and density functional theory to calculate ion flux," $J$. Phys. Cond. Matt., vol. 14, no. 46, pp. 12 129-12 145, 2002.

[37] R. D. Coalson and M. G. Kurnikova, "Poisson-Nernst-Planck theory of ion permeation through biological channels," in Biological Membrane Ion Channels, S.-H. Chung, O. S. Andersen, and V. Krishnamurthy, Eds. New York: Springer, 2007, pp. 449-484.

[38] M. A. Wilson, T. H. Nguyen, and A. Pohorille, "Combining molecular dynamics and an electrodiffusion model to calculate ion channel conductance," J. Chem. Phys., vol. 141, no. 22, 2014.

[39] D. G. Luchinsky, W. A. T. Gibby, I. Kaufman, D. A. Timucin, and P. V. E. McClintock, "Statistical theory of selectivity and conductivity in biological channels," arXiv preprint arXiv:1604.05758, 2016.

[40] P. T. Landsberg, "Einstein and statistical thermodynamics. III. The diffusion-mobility relation in semiconductors," Eur. J. Phys., vol. 2, no. 4 , p. $213,1981$.

[41] I. K. Kaufman, D. G. Luchinsky, R. Tindjong, P. V. E. McClintock, and R. S. Eisenberg, "Multi-ion conduction bands in a simple model of calcium ion channels," Phys. Biol., vol. 10, no. 2, p. 026007, 2013.

[42] D. A. McQuarrie, Statistical Mechanics. Sausalito CA: University Science Books, 2000

[43] A. Parsegian, "Energy of an ion crossing a low dielectric membrane: solutions to four relevant electrostatic problems," Nature, vol. 221, no. 5183, pp. 844-846, 1969.

[44] C. W. J. Beenakker, "Theory of Coulomb-blockade oscillations in the conductance of a quantum dot," Phys. Rev. B, vol. 44, no. 4, pp. 1646$1656,1991$.

[45] D. G. Luchinsky, R. Tindjong, I. K. Kaufman, P. V. E. McClintock, and R. S. Eisenberg, "Self-consistent analytic solution for the current and the access resistance in open ion channels," Phys. Rev. E, vol. 80, no. 2, p. $021925,2009$.

[46] T. Jain, B. C. Rasera, R. J. S. Guerrero, M. S. H. Boutilier, S. C O'Hern, J.-C. Idrobo, and R. Karnik, "Heterogeneous sub-continuum ionic transport in statistically isolated graphene nanopores," Nature Nanotech., vol. 10, no. October, pp. 1-6, 2015. 